\title{
A CLASS OF DISCRETE SPECTRA OF NON-PISOT NUMBERS
}

\section{Dragan Stankov}

\author{
Communicated by Aleksandar Ivić
}

\begin{abstract}
We investigate the class of \pm 1 polynomials evaluated at $q$ defined as:

$$
A(q)=\left\{\epsilon_{0}+\epsilon_{1} q+\cdots+\epsilon_{m} q^{m}: \epsilon_{i} \in\{-1,1\}\right\}
$$

and usually called spectrum, and show that, if $q$ is the root of the polynomial $x^{n}-x^{n-1}-\cdots-x^{k+1}+x^{k}+x^{k-1}+\cdots+x+1$ between 1 and 2 , and $n>2 k+3$, then $A(q)$ is discrete, which means that it does not have any accumulation points.
\end{abstract}

\section{Introduction}

For a real number $q>1$ the spectrum of $q$ is the set of numbers $p(q)$, where $p(x)$ ranges over all polynomial whose coefficients are from a finite set of integers. Here we will consider two spectra

$$
\begin{aligned}
& \Lambda(q):=\left\{\epsilon_{0}+\epsilon_{1} q^{1}+\cdots+\epsilon_{m} q^{m}: \epsilon_{i} \in\{-1,0,1\}\right\}, \\
& A(q):=\left\{\epsilon_{0}+\epsilon_{1} q^{1}+\cdots+\epsilon_{m} q^{m}: \epsilon_{i} \in\{-1,1\}\right\} .
\end{aligned}
$$

Further let us define

$$
\begin{aligned}
l(q) & =\inf \{|y|: y \in \Lambda(q), y \neq 0\}, \\
a(q) & =\inf \{|y|: y \in A(q), y \neq 0\} .
\end{aligned}
$$

It is well known that, if $q$ is a Pisot number, then $\Lambda(q)$ and clearly $A(q)$ are discrete, as a consequence of the Lemma 1.51 in Garsia [5]. (Recall that $q>1$ is called Pisot number if $q$ is an algebraic integer, such that all of the conjugates of $q$, are strictly less than 1 in modulus.) For Pisot numbers satisfying $q^{n}-q^{n-1}-\cdots-q-1=0$, Erdős, Joó and Joó determine that $l(q)=1 / q$ (see [2]). Erdős and Komornik in [3] proved that if $q \in\left(1,2^{1 / 4}\right]$ and if $q^{2}$ is not the second Pisot number, then $l(q)=0$. Whether $\Lambda(q)$ can be discrete for any non-Pisot number is a major open

2000 Mathematics Subject Classification: Primary 11R06; Secondary 11 Y60.

Key words and phrases: Pisot numbers, spectra. 
question in this area. Peres and Solomyak in $[7]$ proved that $A(q)$ is dense for almost every $q \in(\sqrt{2}, 2)$ and asked the same question for $A(q)$. In that case, the question has been answered positively. Borwein and Hare [1] found examples of $q \in(1,2)$ such that $q$ is non-Pisot but $A(q)$ is not dense. The smallest such number found is $\approx 1.72208$, the root of $x^{4}-x^{3}-x^{2}-x+1$, a Salem number. (Recall that $q>1$ is called Salem number if $q$ is a real algebraic integer, such that all of the conjugates of $q$, are less than or equal to 1 in modulus. At least one of the conjugates must be of modulus 1.) Hare in [6] proved that if $q>1$ is a root of the polynomial $x^{n}-x^{n-1}-\cdots-x+1$, then $A(q)$ is discrete. The main result here is the improvement of the Hare's theorem:

THEOREM 1. If $n>2 k+3, k \geqslant 0$ are integers and $q$ is the greatest real root of the polynomial $x^{n}-x^{n-1}-\cdots-x^{k+1}+x^{k}+x^{k-1}+\cdots+x+1$, then $A(q)$ is discrete.

REMARK 1.1. If $n=2 k+3$ then the polynomial can be factored:

$$
\begin{aligned}
x^{2 k+3}-x^{2 k+2}-\cdots-x^{k+1}+x^{k}+x^{k-1} & +\cdots+x+1 \\
& =\left(x^{k+1}-x^{k}-x^{k-1} \cdots-1\right)\left(x^{k+2}-1\right)
\end{aligned}
$$

and the greatest real root of the polynomial is a Pisot number which satisfies $\left(x^{k+1}-x^{k}-x^{k-1} \cdots-1\right)=0$. Clearly, in this case the theorem is still valid. This seems to be the limiting case after which the theorem stops to be a valid one.

\section{Algorithm and the proof}

To prove Theorem 1 we will use a variant of the recursive algorithm of Feng and Wen [4]. We have to modify it to satisfy the hypothesis of the theorem.

For each real number $z$, denote by $T(z)$ the set $\{ \pm q z \pm 1\} \cap[0,1 /(q-1)]$. Let $A_{0}=\{1\}$, recursively we can determine $A_{1}=T(1)$. Suppose that we have determined the set $A_{n}$ with $A_{n} \neq A_{n-1}$, then we obtain the set $A_{n+1}$ by

$$
A_{n+1}=A_{n} \bigcup\left(\bigcup_{z \in A_{n} \backslash A_{n-1}} T(z)\right) \text {. }
$$

If $A_{n}=A_{n-1}$, then the algorithm terminates and we get $A_{n}=A(q) \cap[0,1 /(q-1)]$. We can also conclude that the spectra $A(q)$ are discrete.

Proof of Theorem 1 . To see that $A(q)$ is discrete, simply consider the algorithm and notice that it must terminate. The following observations are needed.

1. $q^{k+1}-q^{k}-q^{k-1}-\cdots-q-1 \geqslant 0$ for $n>2 k+3$.

2. $q^{m}-q^{m-1}-\cdots-q+1>\frac{1}{q-1}$ for all positive integer $m \leqslant n-(k+1)$.

3. $q^{n-l}-q^{n-l-1}-\cdots-q^{k-l+1}+q^{k-l}+\cdots+q-1<-\frac{1}{q-1}$, for $0 \leqslant l \leqslant k$. Thus at each step of the algorithm there is only one choice, and the algorithm must terminate after $n$ steps. It remains to prove these observations.

1. We have supposed that $n>2 k+3$ and the following equality is true:

$$
q^{n}-q^{n-1}-\cdots-q^{k+1}+q^{k}+q^{k-1}+\cdots+q+1=0 .
$$


Adding and subtracting $q^{2 k+3}$ at the left side gives

$\left(q^{n}-q^{n-1}-\cdots-q^{2 k+4}-2 q^{2 k+3}\right)+\left(q^{k+2}-1\right)\left(q^{k+1}-q^{k}-q^{k-1}-\cdots-q-1\right)=0$.

We will show that the first summand $q^{n}-q^{n-1}-\cdots-q^{2 k+4}-2 q^{2 k+3}$ is negative, so we must have $\left(q^{k+2}-1\right)\left(q^{k+1}-q^{k}-q^{k-1}-\cdots-q-1\right)>0$. Here $\left(q^{k+2}-1\right)>0$ finally implies: $q^{k+1}-q^{k}-q^{k-1}-\cdots-q-1>0$.

It remains to prove that in fact $q^{n}-q^{n-1}-\cdots-q^{2 k+4}-2 q^{2 k+3}$ is negative. As we can take the factor $q^{2 k+3}$ outside parentheses $q^{2 k+3}\left(q^{l}-q^{l-1}-\cdots-q-2\right)$, where $l=n-(2 k+3)$, it is sufficient to prove

LEMMA 2.1. $q^{l}-q^{l-1}-\cdots-q-2$ is negative for all positive integers $l$ and for all $q \in(1,2)$.

Proof. The expression in the lemma can be rearranged:

$$
q^{l}-1-\left(q^{l-1}+\cdots+q+1\right)=q^{l}-1-\frac{q^{l}-1}{q-1}=\left(q^{l}-1\right)\left(1-\frac{1}{q-1}\right) .
$$

Now it is clear, as the first factor is positive and the second one is negative, that the product is negative.

2. a) Consider first the case $m \leqslant n-(k+2)$. We need the sequence $q-1$, $q^{2}-q-1, \ldots, q^{n-(k+2)}-q^{n-(k+3)}-\cdots-q-1$. We prove that all the members of the sequence are nonnegative. It is sufficient to show the sequence is decreasing and the last member of it is nonnegative. As the difference of the consequent numbers

$$
q^{m-1}-q^{m-2}-\cdots-q-1-\left(q^{m}-q^{m-1}-\cdots-q-1\right)=q^{m-1}(2-q)>0,
$$

the sequence is decreasing. We assumed that

$$
q^{n}-q^{n-1}-\cdots-q^{k+2}=q^{k+1}-q^{k}-\cdots-q-1
$$

and by dividing this equality by $q^{k+2}$ we get:

$$
q^{n-(k+2)}-q^{n-(k+3)}-\cdots-q-1=\frac{q^{k+1}-q^{k}-\cdots-q-1}{q^{k+2}} \geqslant 0,
$$

using observation 1 , thus the last number of the sequence is nonnegative. We have just proved the next

Lemma 2.2. If the assumptions of Theorem 1 are true and $m \leqslant n-(k+2)$, then $q^{m}-q^{m-1}-\cdots-q-1 \geqslant 0$.

Finally by adding 2 to each side we get $q^{m}-q^{m-1}-\cdots-q+1 \geqslant 2>\frac{1}{q-1}$. The last inequality, that is $2>\frac{1}{q-1}$, follows, as the smallest number of this form is approximately 1.62 .

b) Now consider the case $m=n-(k+1)$. We have to prove that

$$
q^{n-(k+1)}-\cdots-q+1>\frac{1}{q-1} .
$$

Multiplying each side by $q^{k+1}$ one gets an equivalent inequality

$q^{n}-q^{n-1}-\cdots-q^{k+2}-q^{k+1}+q^{k}+\cdots+q+1+\left(2 q^{k+1}-q^{k}-\cdots-q-1\right)>\frac{q^{k+1}}{q-1}$ 
or

Multiplying this by $q-1$ we get

$$
2 q^{k+1}-q^{k}-\cdots-q-1>\frac{q^{k+1}}{q-1} .
$$

$$
2 q^{k+2}-q^{k+1}-\cdots-q^{2}-q-2 q^{k+1}+q^{k}+q^{k-1}+\cdots+1>q^{k+1}
$$

and finally

$$
2 q^{k+2}-4 q^{k+1}+1>0 .
$$

Therefore we have to analyze $P(x)=2 x^{k+2}-4 x^{k+1}+1$. Let us find its approximate values for $x=1,2,2-2^{-(k+2)}$. We can calculate

$$
\begin{gathered}
2 \cdot 1^{k+1}(1-2)+1=-1<0, \quad 2 \cdot 2^{k+1}(2-2)+1=1>0, \\
2\left(2-2^{-(k+2)}\right)^{k+1}\left(2-2^{-(k+2)}-2\right)+1=-\left(1-2^{-(k+3)}\right)^{k+1}+1>0 .
\end{gathered}
$$

We conclude that $P(x)$ has a root $t_{k} \in(1,2)$. Analyzing the sign of the first derivative of $P(x)$ on $[1,2]$ we can see it first decreases, at $x=2-\frac{2}{k+2}$ it has minimum, and then increases. Therefore $t_{k} \in\left(2-\frac{2}{k+2}, 2\right)$ and the polynomial is increasing on this interval. So, if we prove that $q=q_{n, k}>t_{k}$, it would imply $P(q)>P\left(t_{k}\right)=0$ and therefore the inequality (2.2) is true. Then the inequality (2.1) also follows. So let us prove the next

LEMMA 2.3. If $n \geqslant 2 k+4$ and let us denote by $q=q_{n, k}$ the greatest real root of the polynomial

$$
P_{n, k}(x)=x^{n}-x^{n-1}-\cdots-x^{k+1}+x^{k}+x^{k-1}+\cdots+x+1,
$$

and $t=t_{k} \in(1,2)$ the root of the polynomial $P_{k}(x)=2 x^{k+2}-4 x^{k+1}+1$, then $t<q$.

ProOF. First consider the case $n=2 k+4$. The supposition

$$
2 t^{k+2}-4 t^{k+1}+1=0
$$

gives $2 t^{k+2}-4 t^{k+1}+2>0$. Multiplying this by $-1 / 2$ we get $0>-t^{k+3}+2 t^{k+2}-1$ and also $0>-t^{k+3}+\left(2 t^{k+2}+1\right)-2$. The equality $(2.3)$ gives $2 t^{k+2}+1=4 t^{k+1}$. If we replace it in the last inequality we obtain

$$
0>-t^{k+3}+4 t^{k+1}-2 .
$$

The equality (2.3) also gives $-1=2 t^{k+2}-4 t^{k+1}$. Multiplying this by $t^{k+3}$ gives $-t^{k+3}=2 t^{2 k+5}-4 t^{2 k+4}$. If we replace it in the inequality (2.4) we obtain

$$
0>2 t^{2 k+5}-4 t^{2 k+4}+4 t^{k+1}-2 .
$$

Dividing each side of the last inequality by 2 and using $n=2 k+4$ we obtain

$$
\begin{aligned}
0 & >t^{n+1}-2 t^{n}+2 t^{k+1}-1>t^{n+1}-t^{n}-\left(t^{n}-1\right)+2\left(t^{k+1}-1\right) \\
& >t^{n}(t-1)-(t-1)\left(t^{n-1}+t^{n-2}+\cdots+1\right)+2(t-1)\left(t^{k}+t^{k-1}+\cdots+1\right) .
\end{aligned}
$$

Dividing by the positive number $t-1$ gives us

$$
0>t^{n}-\left(t^{n-1}+t^{n-2}+\cdots+1\right)+2\left(t^{k}+t^{k-1}+\cdots+1\right)
$$




$$
0>t^{n}-t^{n-1}-\cdots-t^{k+1}+t^{k}+t^{k-1}+\cdots+t+1 .
$$

As $q_{n, k}$ is the greatest real root of the polynomial, and we have:

$$
\begin{aligned}
& 0=q^{n}-q^{n-1}-\cdots-q^{k+1}+q^{k}+q^{k-1}+\cdots+q+1, \\
& 0<2^{n}-2^{n-1}-\cdots-2^{k+1}+2^{k}+2^{k-1}+\cdots+2+1=2^{k+2}-1,
\end{aligned}
$$

we conclude that $t_{k}<q_{2 k+4, k}<2$.

Now consider the case $n>2 k+4$. We claim that, if $k$ is fixed, the sequence $q_{n, k}$ increases with $n$ and it is bounded by 2 . Indeed,

$$
\begin{aligned}
q_{n, k}^{n+1}-q_{n, k}^{n}-\cdots-q_{n, k}^{k+1}+q_{n, k}^{k}+q_{n, k}^{k-1}+\cdots+q_{n, k}+1 & =q_{n, k}^{n+1}-2 q_{n, k}^{n} \\
& =q_{n, k}^{n}\left(q_{n, k}-2\right)<0,
\end{aligned}
$$

gives $q_{n, k}<q_{n+1, k}$, and

$$
2^{n+1}-2^{n}-\cdots-2^{k+1}+2^{k}+2^{k-1}+\cdots+2+1=2^{k+2}-1>0
$$

gives $q_{n, k}<2$. Finally we conclude that $t_{k}<q_{n, k}$ for all $n \geqslant 2 k+4$.

3. We will need the following

Lemma 2.4. If $-\frac{1}{q-1}<q x+1 \leqslant 0$, and $q \in(1,2)$, then $-\frac{1}{q-1}<x<0$.

Proof. The inequality $q x+1 \leqslant 0$ implies $x \leqslant-\frac{1}{q}<0$ and the other one $-\frac{1}{q-1}<q x+1$ gives $-\frac{1}{q-1}-1<q x$ and $-\frac{q}{q-1}<q x$. Dividing each side by $q$, finaly we get $-\frac{1}{q-1}<x$.

Let us introduce the following finite sequence of the numbers:

$$
\begin{aligned}
\alpha_{n-k-1}= & q^{n-k-1}-q^{n-k-2}-\cdots-q-1, \\
\alpha_{n-k}= & q^{n-k}-q^{n-k-1}-\cdots-q+1, \\
\alpha_{n-k+1}= & q^{n-k+1}-q^{n-k}-\cdots-q^{2}+q+1, \\
& \cdots \\
\alpha_{n}= & q^{n}-q^{n-1}-\cdots-q^{k+1}+q^{k}+\cdots+q+1 .
\end{aligned}
$$

It is easy to verify that $\alpha_{i+1}=q \alpha_{i}+1, i=n-k-1, n-k, \ldots, n-1$. The hypothesis of the theorem gives $\alpha_{n}=0$, so we can use the previous lemma recursively and obtain

$$
\begin{gathered}
-\frac{1}{q-1}<\alpha_{n-1}<0 \\
-\frac{1}{q-1}<\alpha_{n-2}<0 \\
\ldots \\
-\frac{1}{q-1}<\alpha_{n-k-1}<0 .
\end{gathered}
$$


Finally, we have for $0 \leqslant l \leqslant k$

$$
q^{n-l}-q^{n-l-1}-\cdots-q^{k-l+1}+q^{k-l}+\cdots+q-1=\alpha_{n-l}-2 \leqslant-2<-\frac{1}{q-1} .
$$

The last inequality $-2<-\frac{1}{q-1}$ is true because $q>1,62$.

\section{References}

[1] P. Borwein and K. Hare, Some computations on the spectra of Pisot and Salem numbers, Math. Comp. 71 (238) (2002), 767-780.

[2] P. Erdős, I. Joó and M. Joó, On a problem of Tamas Varga, Bull. Soc. Math. France 20 (1992), 507-521.

[3] P. Erdős and V. Komornik, Developments in non-integer bases, Acta Math. Hungar. 79 (1-2) (1998), 57-83.

[4] D.-J. Feng and Z.-Y. Wen, A property of Pisot numbers, J. Number Theory 20(2) (2002), 305-316.

[5] A. M. Garsia, Arithmetic properties of Bernoulli convolutions, Trans. Amer. Math. Soc. 102 (1962), 409-432.

[6] K. G. Hare, Pisot numbers and the spectra of Real numbers, Ph.d. thesis, Simon Fraser University, 2002, June.

[7] Y. Peres and B. Solomyak, Approximation by polynomials with coefficients \pm 1 , J. Number Theory 84 (2) (2000), 185-198.

Katedra matematike RGF-a

(Received 2801 2008)

Univerzitet u Beogradu

Beograd, Đušina 7

Serbia

dstankov@rgf.bg.ac.yu 